\title{
Fatores de risco associados às doenças cardiovasculares em trabalhadores produtores de eucalipto na região do sul da Bahia-Brasil
}

\author{
Risk factors associated with cardiovascular diseases in eucalyptus producing workers in \\ the south region of Bahia-Brazil
}

\section{Factores de riesgo asociados con enfermedades cardiovasculares en trabajadores productores de eucalipto en la región sur de Bahía-Brasil}

Andrea Spier ${ }^{1 *}$, Tátila dos Santos Lima ${ }^{1}$, Lidia Cristina Villela Ribeiro ${ }^{2,3}$, Astria Dias Ferrão Gonzales $^{1,3}$, Djalma Gomes Ferrão Carvalhal ${ }^{3}$, Amanda Catariny de Oliveira Silva ${ }^{4}$, Isa Rita Brito de Morais $^{4}$, Matheus Souza de Moura ${ }^{2}$, Marcos Lázaro da Silva Guerreiro ${ }^{1,2}$.

\section{RESUMO}

Objetivo: Avaliar fatores de riscos associados às doenças cardiovasculares e qualidade de vida em trabalhadores da eucaliptocultura por meio de informações sociodemográficas, do consumo dietético e medidas antropométricas. Métodos: $O$ estudo é de natureza descritiva, transversal, quantiqualitativo no qual foram avaliados 70 trabalhadores do sexo masculino, em uma empresa privada no Extremo Sul da Bahia. Para coleta de informações utilizou-se a entrevista por meio de questionário, onde se investigou medidas de peso, estatura, e circunferência da cintura e índice de massa e gordura corporal. Para análise do consumo dietético utilizou-se o recordatório de 24horas e o Questionário de Frequência Alimentar (QFA). Os dados obtidos foram analisados por meio da estatística descritiva com auxílio do Programa Excel 2010. Resultados: Os trabalhadores apresentam média de idade de 46,8 anos, $85,7 \%$ com primeiro grau incompleto, $64 \%$ em situação de Pré-Hipertensão, 15,7\% são portadores de Hipertensão Arterial Sistêmica e 38,57\% encontramse com sobrepeso. A maioria não faz atividade física e a dieta consumida é composta por baixa ingestão de alimentos cardioprotetores. Conclusão: $O$ grupo estudado apresenta fatores sociodemográficos, composição corporal e consumo alimentar que expressa risco eminente para doenças cardiovasculares necessitando de intervenção e acompanhamento nutricional.

Palavras-chave: Eucaliptocultura, Silvicultores, Consumo alimentar, Doenças cardiovasculares.

\begin{abstract}
Objective: To assess risk factors associated with cardiovascular disease and quality of life in eucalyptus culture workers through socio-demographic information, dietary intake and anthropometric measures. Methods: The study is descriptive, cross-sectional, quantitative and qualitative in which 70 male workers were evaluated in a private company in the extreme south of Bahia. To collect information, the interview was used by means of a questionnaire, which investigated measures of weight, height, and waist circumference and body mass and fat index. For the analysis of dietary consumption, the 24-hour recall and the Food Frequency Questionnaire (FFQ) were used. The data obtained were analyzed using descriptive statistics with the help of the Excel 2010 program. Results: Workers have a mean age of 46.8 years, $85.7 \%$ with incomplete primary education, $64 \%$ in pre-hypertension situation, 15, 7\% have Systemic Arterial Hypertension and $38.57 \%$ are overweight. Most do not do physical activity and the diet consumed consists of low intake of cardioprotective foods. Conclusion: The studied group presents sociodemographic factors, body composition and food consumption that expresses eminent risk for cardiovascular diseases requiring nutritional intervention and monitoring.
\end{abstract}

Key words: Eucalyptus culture, Silviculture, Food consumption, Cardiovascular diseases.

\footnotetext{
${ }^{1}$ Centro Universitário UniFTC, Salvador - BA. *E-mail: aspier.ita@ftc.edu.br

2 Universidade Estadual de Feira de Santana (UEFS), Feira de Santana - BA.

3 Universidade do Estado da Bahia (UNEB), Salvador - BA.

${ }^{4}$ Universidade Federal da Bahia (UFBA), Salvador - BA.
} 


\section{RESUMEN}

Objetivo: Evaluar factores de riesgo asociados a enfermedades cardiovasculares y calidad de vida en trabajadores del cultivo de eucalipto a través de información sociodemográfica, ingesta dietética y medidas antropométricas. Métodos: Se trata de un estudio descriptivo, transversal y cuantitativo en el que se evaluaron 70 trabajadores del sexo masculino de una empresa privada del extremo sur de Bahía. Para la recolección de información se utilizó la entrevista mediante un cuestionario, medidas de peso, talla y circunferencias, y se utilizó el recordatorio de 24 horas y el Cuestionario de Frecuencia Alimentaria para analizar el consumo dietético. La información fue obtenida por los investigadores con CEP 2.513.272 / FTC, y los datos analizados mediante estadística descriptiva con la ayuda del programa Excel 2010. Resultados: El público tiene una edad promedio de 46,8 años $\pm 11,2$ años, $85,7 \%$ con datos incompletos. primer grado, $15,7 \%$ tiene HAS; $64 \%$ tenía prehipertensión; $47 \%$ sobrepeso, la mayoría no realiza actividad física y la dieta consumida da una baja ingesta de alimentos cardioprotectores. Conclusión: El grupo estudiado presenta factores sociodemográficos, composición corporal y consumo de alimentos que expresan un riesgo eminente de enfermedades cardiovasculares que requieren intervención nutricional y seguimiento nutricional.

Palabras clave: Cultivo de eucalipto, Silvicultura, Consumo de alimentos, Enfermedades cardiovasculares.

\section{INTRODUÇÃO}

A biomassa do eucalipto tem sido utilizada como fonte de energia produzida em larga escala em diversos países na busca de atender o gasto energético da indústria siderúrgica, porém atrelada às preocupações por uma produção de energia mais limpa e sustentável. Para atender essa alta demanda energética, o setor florestal tem nos últimos anos empregado milhares de trabalhadores (SANTOS SFOM e HATAKEYAMA K, 2012). Segundo Nascimento KAO, et al., (2018), o trabalho braçal no manejo do eucalipto envolve um gasto energético elevado, necessitando assim, de um maior aporte nutricional.

Lima TS, et al (2020) avaliando o perfil nutricional em silvicultores constatou que a dieta desses trabalhadores era composta pela alta ingestão de carboidratos e alimentos inflamatórios por mais de cinco dias durante a semana, constituindo-se o principal fator de risco para o surgimento de diabetes e doenças cardiovasculares.

Em 2017, as doenças cardiovasculares foram a principal causa de morte no mundo, representando $31 \%$ de todas as mortes no cenário global em 2015, sendo na contemporaneidade as enfermidades que mais matam em relação a qualquer outra causa (OPAS/OMS, 2017). Em 2019, 5,3\% (8,4 milhões) de pessoas com 18 anos ou mais foram diagnosticadas com alguma doença do aparelho circulatório, sendo estas as principais causas de morte no país, ocasionando os maiores custos em internações hospitalares em todo o território nacional (IBGE, 2019).

Dentre os fatores de risco que estão associados às Doenças Cardiovasculares, estão a ingesta alimentar, a hipertensão, obesidade, tabagismo, consumo abusivo de álcool, inatividade física, fatores socioeconômicos e ambientais (PRÉCOMA DB, 2019). No que tange à alimentação, padrões alimentares mais saudáveis têm sido recomendados na prevenção da DCV em diversos países, uma vez que, não se deve valorizar um único padrão alimentar, e sim, distribuir e equilibrar às refeições considerando a qualidade e quantidade dos alimentos a serem consumidos durante o dia, algo não observado na presente população avaliada.

A associação entre as diferentes ocupações e as doenças cardiovasculares, já são bem documentadas na literatura. Segundo Wada K, et al. (2016) algumas ocupações como as relacionadas à agricultura, pesca, construção civil e mineração apresentam maiores riscos de desfechos cardiovasculares como doenças isquêmicas do coração. Outros estudos apontam, ainda, alguns fatores que poderiam predizer um maior risco de problemas cardiovasculares como, por exemplo, o número de horas trabalhadas, sedentarismo, altos consomem de bebidas alcoólicas e a ingesta alimentar errônea durante sua jornada laboral (CAIRES MCB, et al., 2021; KIVIMÄKI M, et al., 2015; SMITH P, et al., 2017).

Como forma de prevenir as DCVs, deve-se incentivar um consumo calórico com predomínio de cereais fibrosos, frutas e hortaliças, com restrição de carboidratos refinados, embutidos e alimentos ultra processados, 
priorizando os ácidos graxos poli-insaturados, gorduras mais saudáveis em detrimento das saturadas e trans (IZAR MCO, 2021).

Nesse tocante, avaliar a dieta em silvicultores, permiti identificar quais fatores podem influenciar diretamente na saúde dos trabalhadores pertencentes a esse grupo e a outros grupos similares, como por exemplo: o tabagismo, a composição corporal e o tipo de alimentação que contribui ou preveni a gênese das doenças cardiovasculares (LIMA TS, et al., 2020)

Desta forma, objetivou-se investigar a presença de fatores de riscos associados às doenças cardiovasculares em trabalhadores de uma empresa prestadora de serviços na silvicultura, na Região do Estado Bahia.

\section{MÉTODOS}

Este é um estudo observacional, descritivo, transversal, quantitativo, que compreende uma amostra não probabilística, realizada em trabalhadores que vivem exclusivamente da silvicultura. O presente estudo foi realizado com trabalhadores de uma empresa privada produtora de eucalipto no Extremo Sul da Bahia, que presta serviços florestais (manejo, corte e reflorestamento) para a produção de eucalipto.

O estudo contou com uma amostra de 70 funcionários em regime celetista com no mínimo de seis meses carteira assinada nesta empresa, e que exercessem atividades relacionadas ao trabalho manual da silvicultura como o roceamento, coveamento, aplicação de defensivos, coroamento e plantio de eucalipto, sendo exclusos da amostra todos os outros funcionários, que exercessem atividades semi-mecanizadas e ou mecanizadas. O Termo de Consentimento Livre e Esclarecido (TCLE) foi explicado aos trabalhadores e assinado pelos mesmos. A pesquisa obedece à resolução 466/12 tendo aprovação do CEP/Centro Universitário-UniFTC sob ํㅜㄹ 2.513.272. Para coleta de informações foram utilizadas entrevistas estruturadas fechadas através de questionários aplicados pelo grupo de pesquisadores.

A avaliação antropométrica foi realizada utilizando as medidas de peso, estatura e circunferência da cintura (CC), seguindo os protocolos de acordo com BRASIL (2013). Desta forma, a medida do peso foi aferida em balança portátil digital, com medidor de altura digital marca WISO, modelo W721 com capacidade de $180 \mathrm{~kg}$, graduada de 100 em 100 gramas. A medida da CC foi realizada com fita resistente, inelástica, porém flexível da marca Sanny®, com precisão de $0,1 \mathrm{~cm}$ tendo no total $150 \mathrm{~cm}$, no ponto médio entre a borda inferior da última costela e o osso do quadril (crista ilíaca), no momento da expiração. Para circunferência do braço foi tomada a medida do ponto médio entre o acrômio e olecrano com o braço flexionado em direção ao tórax, formando um ângulo de 90ㅜ logo foi aferida a circunferência com o braço relaxado, sendo avaliada por meio de percentis (FRISANCHO AR, 1990; BLACKBURN GL e THORNTON PA, 1979).

O consumo alimentar foi avaliado por meio do registro de 24 horas (Recordatório) e Questionário de Frequência Alimentar. O Valor Energético Total (VET) das refeições, total de macronutrientes e micronutrientes, bem como, o cálculo do Gasto Energético Diário (GET) foram computados por meio de Softwear Nutricional DietSmartß. Para análise de consumo de macro e micronutrientes considerou-se as recomendações das Dietary References Intakes (DRI's, 2011).

Ressalta-se que para cálculo do GET dos trabalhadores foi utilizado o método do equivalente Metabólico da tarefa (MET's) proposto por Ainsworth, BE et al. (2011) que contempla todas as atividades diárias das 24horas, incluindo o equivalente metabólico específico para atividade de manejo florestal.

A pressão Arterial (PA) dos trabalhadores foi aferida, por meio de aparelho de pressão digital automático de braço da marca G.TECH modelo MA 100, respeitando o protocolo para aferição segundo as recomendações da SBC (2016). Foram realizadas a média de duas aferições antes dos trabalhadores iniciarem o trabalho, sendo considerado os valores de Hipertensão Arterial Sistêmica (HAS) a Pressão Sistólica $\geq 141 \mathrm{mmHg}$ e Pressão Diastólica $\geq 91 \mathrm{mmHg}$. Já a Pré-Hipertensão é denominada como a Pressão Sistólica $\geq 121 \mathrm{mmHg}$ e Pressão Diastólica $\geq 81 \mathrm{mmHg}$, sendo esta uma nova classificação proposta pela Sociedade Brasileira de Cardiologia - SBC (2016). 
Considerou-se como atividade física a prática de no mínimo 30min/dia no modo aeróbico, por pelo menos 3 vezes na semana visando a prevenção segundo orientação da Diretriz de Prevenção Cardiovascular da Sociedade Brasileira de Cardiologia (SBC, 2019).

Em relação ao consumo de bebidas alcoólicas foi considerado o consumo diário de no máximo 2 latas $(350 \times 2=700 \mathrm{ml})$ ou 1 garrafa $(650 \mathrm{ml})$ de cerveja; 2 taças de $150 \mathrm{ml}$ ou 1 taça de $300 \mathrm{ml}$ de vinho; 2 doses de $50 \mathrm{ml}$ de uísque, vodca ou bebida destilada, conforme Diretriz de Prevenção Cardiovascular da Sociedade Brasileira de Cardiologia (SBC, 2019).

Todos os dados foram tabulados e analisados no programa Excel 2010. Os dados contínuos não paramétricos foram categorizados a partir da mediana e apresentados na forma de frequência absoluta (n) e frequência relativa (\%). As médias foram calculadas verificando-se o coeficiente de variação.

\section{RESULTADOS E DISCUSSÃO}

Os participantes da pesquisa totalizaram 70 funcionários, todos do sexo masculino variando entre 21 a 73 anos, no qual predominaram as faixas etárias entre 41 a 50 anos $(27,1 \%)$ e 51 a 60 anos (40\%), de etnia parda (64,3\%), conforme a Tabela 1. Em relação à idade, para Picon RV (2013), o fator etário é considerado fator de risco conhecido por haver uma associação direta entre o aumento da idade e a prevalência de Hipertensão Arterial (HA), fator este que é atribuído principalmente pelo aumento da expectativa de vida e da população de idosos no país.

Quanto à etnia os estudos são unânimes ao apresentarem a etnia negra como prevalente quando comparados às outras (IBGE, 2019). Um estudo relacionando o tratamento, condições socioeconômicas e etnias, demonstrou uma prevalência de 49,3\% em negros em relação às outras etnias, dados que corroboram com nossos achados, pois, a cor parda pertence à etnia negra (CHOR D, 2015).

Tabela 1 - Características sociodemográficas de trabalhadores florestais em atividade manual de uma empresa prestadora de serviço, Região Sul da Bahia, 2018.

\begin{tabular}{|c|c|c|c|}
\hline Variáveis & Trabalhador (und.) & Trabalhador (\%) & Média (DP) \\
\hline \multicolumn{4}{|l|}{ Sexo } \\
\hline Masculino & 70 & 100,0 & \\
\hline Faixa etária & & & $46,8(11,2)$ \\
\hline 21 a 30 anos & 6 & 8,6 & \\
\hline 31 a 40 anos & 12 & 17,1 & \\
\hline 41 a 50 anos & 19 & 27,1 & \\
\hline 51 a 60 anos & 28 & 40 & \\
\hline$>61$ anos & 5 & 7,1 & \\
\hline \multicolumn{4}{|l|}{ Cor da pele } \\
\hline Negro & 11 & 15,7 & \\
\hline Branco & 14 & 20 & \\
\hline Pardo & 45 & 64,3 & \\
\hline Amarelo & 0 & 0 & \\
\hline \multicolumn{4}{|l|}{ Escolaridade } \\
\hline Sem nenhuma instrução formal & 7 & 10 & \\
\hline 1ํograu incompleto/semialfabetizado & 60 & 85,7 & \\
\hline 1ํgrau completo & 2 & 2,9 & \\
\hline $2^{\circ}$ grau incompleto & 0 & 0 & \\
\hline 2ํgrau completo & 1 & 1,4 & \\
\hline \multicolumn{4}{|l|}{ Renda Mensal } \\
\hline Até 1 salário mínimo & 70 & 100,0 & \\
\hline >1 salário mínimo & 0 & 0 & \\
\hline Total & 70 & 100 & \\
\hline
\end{tabular}

Legenda: Valores apresentados em (\%) ou media \pm DP.

Fonte: Spier A, et al. 2020. 
Em relação ao nível de instrução, $85,7 \%$ dos trabalhadores da pesquisa não possuem o ensino fundamental completo o que os inserem em um público com maiores chances de adquirirem Hipertensão Arterial Sistêmica (HAS), pois dados da PNS revelaram que 31,1\% das pessoas sem instrução ou com ensino fundamental incompleto referiram ser portadoras de HAS, havendo uma relação inversamente proporcional de HAS com o nível de instrução (IBGE, 2019).

Além do fator instrução (escolaridade) o aspecto socioeconômico parece relevante tendo em vista que os trabalhadores desta pesquisa, em sua grande maioria, recebem um salário mínimo independente do tempo de trabalho na empresa. Gaudemaris R, et al. (2002), ao estudarem em um grupo populacional francês identificaram maior prevalência da pressão arterial entre os trabalhadores com menor faixa de renda, além da maior dificuldade para a manutenção do controle terapêutico da PA.

Os dados encontrados referentes ao hábito de fumar e consumo de bebidas alcoólicas corroboram com o perfil de trabalhadores florestais estudados por Lima TS, et al. (2020), no qual encontrou dados semelhantes na população estudada. Os dados referentes ao estilo de vida dos trabalhadores podem ser vistos na Tabela 2.

Tabela 2 - Fatores de risco no estilo de vida de trabalhadores florestais em atividade manual de uma empresa prestadora de serviço na Região Sul da Bahia, 2018.

\begin{tabular}{lcc}
\hline Variáveis & Trabalhador (und.) & Trabalhador (\%) \\
\hline Prática de atividades físicas & & \\
\hline Sim & 15 & 21,4 \\
Não & 55 & 78,6 \\
\hline Tabagismo & 9 & 12,9 \\
\hline Sim & 61 & 87,1 \\
Não & & \\
\hline Uso habitual de bebidas alcoólicas & 26 & 37,1 \\
\hline Sim & 44 & 62,9 \\
Não & & \\
\hline Diabetes Mellitus & 1 & 1,42 \\
\hline Sim & 69 & 98,6 \\
Não & & 15,7 \\
\hline Hipertensão Arterial Sistêmica & 11 & \\
\hline Sim & 59 & 64,3 \\
Não & & 36 \\
\hline Pré-Hipertensão & 45 & $\mathbf{1 0 0}$ \\
\hline Sim & 25 & \\
Não & 70 & \\
\hline Total & & \\
\hline
\end{tabular}

Legenda: Valores apresentados em (\%).

Fonte: Spier A, et al. 2020.

Ressalta-se que mesmo sendo a minoria dos trabalhadores, autodeclarados tabagistas, a adoção de estratégias preventivas precoces e controle no comportamento do tabagismo são necessárias, visto que para Yun M, et al. (2015) ao avaliarem 945 adultos (dos 23 aos 43 anos) que tiveram a PA medida desde a infância (4 a 17 anos) foi detectado que fumar foi um preditor significativo para enrijecimento arterial.

A prática de atividade física da maior parte dos trabalhadores esteve relacionada ao ir e vir do trabalho para casa de bicicleta. Para o IBGE (2019) o sedentarismo é preocupante, uma vez que há uma forte correlação com a obesidade devido à falta regular de exercícios e bons hábitos de vida. Esses fatores em conjunto, propiciam um aumento significativo das chances de se desenvolver outras doenças crônicas associadas. 
Os trabalhadores avaliados perfazem um grupo com 47,11\% de excesso de peso, em relação ao IMC. Esses valores corroboram com o estudo de Souza E, et al. (2012) no qual avaliaram um grupo de trabalhadores rurais, cortadores de cana-de-açúcar, no qual foi encontrada uma prevalência de $46 \%$ de excesso de peso. No nosso estudo, os itens sobre peso e obesidade somam na população estudada $47,11 \%$, dados que sugerem eminência de alto risco no desenvolvimento de doenças cardiovasculares. Esses achados são aferidos pela análise dos dados da composição corporal e estão apresentados na Tabela 3.

Tabela 3 - Dados antropométricos avaliados em trabalhadores florestais de uma empresa prestadora de serviço na Região Sul da Bahia, 2018.

\begin{tabular}{lccc}
\hline Variáveis & Trabalhador (und.) & Trabalhador (\%) & Média (DP) \\
\hline Índice de Massa Corporal (IMC) & & & \\
\hline Magreza & 56 & 31,4 & \\
Eutrofia & 27 & 38,57 & $25,21(3,52)$ \\
Sobrepeso & 6 & 8,54 & \\
Obesidade & & & 89, 17 (10,32) \\
\hline Circunferência da Cintura (CC) & 7 & 10 & \\
\hline Risco & 9 & 12.8 & \\
Alto risco & 54 & 77,2 & \\
Sem risco & & & \\
\hline \% de Gordura Corporal & 0 & 7,1 & \\
\hline Abaixo da média & 5 & 45,7 & \\
Na média & 32 & 47,1 & \\
Acima da média & 33 & $\mathbf{1 0 0} \%$ & \\
Risco de doenças associadas à obesidade & $\mathbf{7 0}$ & & \\
\hline Total & & & \\
\hline
\end{tabular}

Legenda: Valores apresentados em (\%) ou media \pm DP.

Fonte: Spier A, et al., 2020.

Ao se verificar o risco de desenvolver DCV por meio da circunferência da cintura, os trabalhadores avaliados apresentaram risco e alto risco de $22,8 \%$. O estudo de Souza E, et al. (2012) verificou $90 \%$ (risco) e $84,3 \%$ (alto risco), valores muito acima dos encontrados em nosso estudo. Entretanto, nossos achados são importantes e pertinentes pois, apontam riscos eminentes para 0 desenvolvimento de doenças cardiovasculares a longo prazo.

Ressalta-se que o IMC apesar de não diferenciar os compartimentos corporais no que diz respeito à fração de gordura e músculo, é um índice que apresenta alta correlação com a gordura corporal e consequentemente com comorbidades (SAMPAIO LR, 2012). Em nosso estudo, por estarmos avaliando um público que exerce atividade de força, optou-se também por avaliar o percentual de gordura $(\% \mathrm{G})$ desses trabalhadores que revelou $92,8 \%$ acima da média esperada. Esses achados revelam que uma atividade de força física quando não padronizada na forma de exercícios físicos e dieta equilibrada, não contribuem para a manutenção do peso corporal estimado pelo IMC.

Em relação ao consumo dos alimentos designados de risco para o desenvolvimento de DCV, é demonstrado que o consumo de cereais refinados, carnes com gorduras e açúcares aparecem em seis ou mais dias. Os embutidos conferem um consumo de três até cinco dias na semana, visto que são alimentos que compõe parte de das refeições fornecidas pela empresa, Tabela 4. 
Tabela 4 - Frequência do consumo semanal de alimentos protetores e de risco para DCV de acordo com o Questionário de Frequência Alimentar (QFA) aplicado aos trabalhadores florestais, em atividade manual, na Região Sul da Bahia, 2018.

\begin{tabular}{|c|c|c|c|}
\hline \multirow{2}{*}{$\begin{array}{l}\text { Variáveis } \\
\text { Grupos alimentares }\end{array}$} & \multicolumn{3}{|c|}{ Frequência Semanal } \\
\hline & $\leq 2$ dias & $\geq 3$ a $\leq 5$ dias & $\geq 6$ dias \\
\hline Alimentos protetores & n (\%) & n (\%) & n (\%) \\
\hline Salada/folhosos & $39(55,7)$ & $20(28,5)$ & $11(15,7)$ \\
\hline Legumes $^{a}$ & $51(72,8)$ & $12(17,1)$ & $7(10)$ \\
\hline Cereais Integrais $^{b}$ & $65(92,8)$ & $3(4,2)$ & $2(2,8)$ \\
\hline Frutas & $39(55,7)$ & $19(27,1)$ & $12(17,1)$ \\
\hline Feijão & - & - & $70(100)$ \\
\hline Carnes sem gordura & $70(100)$ & - & - \\
\hline Peixes & $69(98,5)$ & $1(1,4)$ & - \\
\hline Leite desnatado & $70(100)$ & - & - \\
\hline Ovos & $62(88,5)$ & $7(10)$ & $1(1,4)$ \\
\hline Chá & $38(54,2)$ & $22(31,4)$ & $10(14,2)$ \\
\hline \multicolumn{4}{|l|}{ Alimentos de Risco } \\
\hline Cereais Refinados ${ }^{c}$ & - & - & $70(100)$ \\
\hline Carnes com gordura & - & - & $70(100)$ \\
\hline Leite Integral & $21(30)$ & $9(12,8)$ & $40(57,1)$ \\
\hline Lanches fritos $^{d}$ & $70(100)$ & - & - \\
\hline Embutidos & - & $65(92,4)$ & $5(7,1)$ \\
\hline Gordura/sódioe & $35(50)$ & $15(21,4)$ & $20(28,5)$ \\
\hline Açúcares ${ }^{f}$ & $1(1,4)$ & - & $69(98,5)$ \\
\hline Bebida alcóolica & $66(94,2)$ & - & $4(5,7)$ \\
\hline
\end{tabular}

Legenda: Valores exibidos em (\%) da frequência semanal de alimentos consumidos pelos participantes do estudo. a Cenoura, abóbora, chuchu, brócolis, abobrinha e quiabo. ${ }^{b}$ Raízes e tubérculos. ${ }^{c}$ Pães, massas em geral, macarrão, biscoito e arroz branco. ${ }^{d}$ Batata frita, nuggets, batata chips, salgado frito (coxinha, quibe, pastel). e Bolachas e biscoitos. ${ }^{\dagger}$ Refrigerante, suco artificial, biscoito recheado, balas e chocolates, açúcar branco. - Não consome.

Fonte: Spier A, et al., 2020.

Poucos estudos publicados em trabalhadores florestais avaliaram o consumo alimentar desse público no que diz respeito aos aspectos qualitativos da dieta Luz TC, at al. (2020). Segundo a SBC (2013), os alimentos protetores para a prevenção de DCV são as frutas, vegetais, laticínios na versão desnatada, precursores de fibras e minerais como cálcio e potássio. Observamos que à medida que o consumo semanal dos alimentos de risco aumenta, os considerados protetores são reduzidos, exceto o feijão que aparece seis dias na semana.

O consumo dos alimentos protetores ricos em antioxidantes como as frutas, legumes e vegetais (FLV) é importante, visto que oferecem proteção ao organismo. Commerer MA, et al. (2018), avaliaram trinta e três pacientes com risco cardiovascular por meio de marcadores inflamatórios e perfil lipídico com dois tipos de dieta. A primeira, antioxidante rica em flavonoides (contendo vegetais crucíferos, tomate cereja, cebola, e frutas como laranja, maçã, uvas roxas) e a segunda, dieta específica para redução de colesterol adaptado de National Cholesterol Education Program (NCEP) Adult Treatment Panel (ATP) III recommendations.

Os pacientes foram acompanhados por seis meses e ao final, não foi verificado redução nos fatores inflamatórios. Entretanto, o grupo de intervenção que seguiu uma dieta rica em antioxidantes obteve redução no LDL- colesterol, indicando benefícios cardiovasculares apesar da inflamação e dos marcadores do estresse oxidativo. 
O estudo conduzido por Chen MU, et al. (2016), avaliou a associação da gordura láctea com a DCV em trabalhadores adultos norte-americanos (homens e mulheres). Estes autores observaram que a substituição de $5 \%$ de gordura láctea por ácido graxo poli-insaturado ou gordura vegetal levou a uma redução de risco de DCV de $24 \%$ em homens e $10 \%$ em mulheres. No entanto, a substituição de $5 \%$ da ingestão de outras gorduras animais por gordura láctea foi associada ao aumento de $6 \%$ de risco de DCV. Por lado, quando houve a substituição da gordura láctea por carboidratos, com fonte nos grãos integrais, observaram menor risco.

Augustin LSA, et al. (2015), em um consenso científico internacional sobre quantidade e qualidade dos carboidratos da dieta, chamam a atenção para o fato de que os alimentos com alto índice glicêmico e pobres em fibras proporcionam elevação da glicose sanguínea gerando picos de insulina que podem desencadear resistência a este hormônio, levando à obesidade e ao risco para doenças cardiovasculares.

De acordo com nossos resultados, a dieta de carboidratos no percentual 68,37 ultrapassam a recomendação referida pelas DRI's (2011), o que se justifica por ser um grupo de alimentos que faz parte da rotina dos trabalhadores como consta na Tabela 5.

Tabela 5 - Dados do consumo de macronutrientes, perfil lipídico, fibras e minerais (sódio e potássio), por trabalhadores pertencentes à amostra do estudo.

\begin{tabular}{|c|c|c|}
\hline Consumo de Macronutrientes & Consumo \% & Recomendações DRIs (2011) \\
\hline Carboidratos & $68,37 \%$ & 45 a $65 \%$ \\
\hline Proteínas & $14,67 \%$ & 10 a $35 \%$ \\
\hline Lipídios & $16,96 \%$ & 20 a $35 \%$ \\
\hline Ácidos Graxos Saturados (AGS) & $25,8 \mathrm{~g}(6,52 \%)$ & $\begin{array}{c}\text { sem comorbidade } \leq 10 \% \\
\text { com comorbidade } \leq 7 \%\end{array}$ \\
\hline Ácidos Graxos Monoinsaturados (AGM) & $27,35 \mathrm{~g}(6,91 \%)$ & $15 \%$ \\
\hline Ácidos Graxos Poli-insaturados (AGPI) & $12,23 \mathrm{~g}(3,9 \%)$ & $5-10 \%$ \\
\hline Colesterol & $248,12 \mathrm{mg}$ & Recomendação não estabelecida \\
\hline Fibras & $48,07 \%$ & $\begin{array}{l}38 \mathrm{~g}(19 \text { a } 50 \text { anos }) \\
30 \mathrm{~g}(51-70 \text { anos })\end{array}$ \\
\hline Sódio & $2.484,5 \mathrm{mg}$ & $\begin{array}{c}1500 \mathrm{mg} \text { (31 - } 50 \text { anos) } \\
1300 \mathrm{mg}(51-70 \text { anos }) \\
2000 \mathrm{mg}(\mathrm{SBC}, 2013)\end{array}$ \\
\hline Potássio & $3.313,3$ & $4.700 \mathrm{mg}$ \\
\hline Água & $2.950 \mathrm{~mL}$ & $5.000 \mathrm{~L}$ \\
\hline
\end{tabular}

Legenda: Dados do Recordatório de 24 horas, aplicado aos trabalhadores florestais em 2018.

Fonte: Spier A, et al. 2020.

Em relação ao perfil lipídico da dieta consumida, os ácidos graxos saturados (AGS) são mais consumidos contrariando os índices recomendados. Entretanto, os AGM e AGPI considerados protetores para DCV ficaram abaixo dos valores aconselhados. Para o colesterol total da dieta, a última Diretriz Brasileira de Dislipidemia e Prevenção de Aterosclerose (SBC 2013) refere que não há evidencias suficientes para o estabelecimento de um valor de referência, visto que os últimos estudos, não apontam influência direta na mortalidade, pois depende de fatores intrínsecos de cada indivíduo.

Em relação à especificidade de prevenção de placas ateroscleróticas, Mateo-Gallego R, et al. (2017), avaliaram a relação da adesão à Dieta Mediterrânea (Dmedi) rica em FLV, carnes brancas, grãos integrais e azeite com a presença de placas ateroscleróticas em 2588 trabalhadores de meia-idade 51,3 anos $( \pm 3,89)$. Estes foram submetidos a ultrassom em carótida, femoral e aorta para quantificação e espessura das placas. Observaram que quanto maior a adesão à Dmedi, menor a presença de placas, número e espessura das mesmas, sendo uma associação mais forte para as artérias femorais e entre os fumantes. Esses dados demonstram que a dieta dos trabalhadores investigada no presente estudo precisa de uma intervenção o mais rápido possível a fim de reduzir os riscos. 
Já o consumo de fibra total da dieta perfez um total de $48,07 \mathrm{~g}$, o que pode ser justificado pelo alto consumo do feijão e não de outras fontes alimentares como as frutas, legumes e vegetais. A metanálise de Khan $\mathrm{K}$, et al. (2018) revelou que o uso de $8,7 \mathrm{~g}$ de fibras solúveis na dieta, utilizados durante sete semanas, reduziu as pressões sistólica e diastólica dos indivíduos avaliados, diminuindo o risco para DCV.

O consumo de sódio ultrapassa as recomendações e o consumo de potássio encontra-se abaixo do recomendado o que pode ser atribuído ao consumo de embutidos e uma dieta pobre em alimentos fontes desse mineral, como as frutas e os legumes, respectivamente, conforme a Tabela 5. Em estudo realizado por Porto AS, et al. (2014), foi encontrado que o consumo de sódio por indivíduos adultos encontrava-se acima das recomendações previstas e o consumo de potássio abaixo, incidindo em risco para eventos cardiovasculares. Os mesmos autores afirmam também que incentivar a escolha de alimentos ricos em potássio como feijões, vegetais verdes e frutas pode ser uma alternativa eficiente para o controle e prevenção de DCV.

A média de água consumida foi de $2.950 \mathrm{~mL} / \mathrm{dia}$, um valor abaixo das necessidades recomendadas pelo Guia Alimentar da População Brasileira (BRASIL, 2019) o qual estabelece $1 \mathrm{~mL}$ por energia (Kcal) gasta. Considerando que o GET médio foi de $4.884,64 \mathrm{Kcal}( \pm 709,80 \mathrm{Kcal})$ o valor recomendado seria em torno de $5 \mathrm{~L}$ de água/dia. Para Coelho MP, et al. (2016) as condições de trabalho a céu aberto contribuem para o excesso de calor corporal, predispondo à fadiga com redução da produtividade e aumento dos riscos de acidentes no trabalho. Desta forma, o autor propõe implementar estratégias de reposição hídrica e de sais minerais apropriadas às demandas desses trabalhadores. Esta recomendação foi discutida e recomendada aos participantes deste estudo.

O tratamento dietético na prevenção das DCV preconiza o consumo de determinados alimentos vistos como protetores, na medida em que estudos apontam que outros alimentos exercem ação pró-inflamatória. Assim, um padrão alimentar mais do que a ingestão de um ou outro alimento específico, deverá ser composto de alimentos saudáveis já que ocorre um sinergismo entre os nutrientes e seus compostos bioativos, favorecendo a modulação da inflamação arterial e reduzindo os riscos da DCV (GARCÍA-ARELLANO A, et al., 2015).

\section{CONCLUSÂO}

Neste estudo, constata-se a necessidade mudanças estruturais relacionadas ao cardápio, à dieta e ao acompanhamento nutricional na população estudada, já que fatores como: qualidade de vida, perfil antropométrico e consumo alimentar nesses trabalhadores expressam riscos eminentes relacionados ao desenvolvimento das doenças cardiovasculares. Assim, a adequação no cardápio oferecido e a realização de atividades em grupo, com foco na educação alimentar e nutricional, propiciará autonomia nas escolhas alimentares saudáveis, favorecendo um maior consumo de alimentos considerados protetores para a saúde cardiovascular. As limitações da investigação realizada deram-se pela impossibilidade de realizar exames mais complexos nos sujeitos da pesquisa.

\section{REFERÊNCIAS}

1. AINSWORTH BE, et al. Compendium of Physical Activities: A Second Update of Codes and MET Values. Med Sci Sports Exerc, 2011; 43(8): 1575-812.

2. AUGUSTIN LSA, et al. Glycemic index, glycemic load and glycemic response: An International Scientific Consensus Summit from the International Carbohydrate Quality Consortium (ICQC). Nutrition, Metabolism \& Cardiovascular Diseases, 2015; 24: 795-815.

3. BLACKBURN GL, e THORNTON PA. Nutritional assessment of the hospitalized patients. Med. Clin. North Am, 1979; (63)1103-1115.

4. BRASIL. Secretaria de atenção à saúde. Coordenação-geral da Política de Alimentação e Nutrição. 2019. Guia Alimentar para população brasileira: promovendo a alimentação saudável. Brasília: Ministério da Saúde,

5. BRASIL. Ministério da Saúde. Secretaria de Atenção à Saúde. Política Nacional de Alimentação e Nutrição / Ministério da Saúde. 1. ed., 1. Reimpr. 2013; 84: 85-334.

6. CHEN MU, et al. Dairy fat and risk of cardiovascular disease in 3 cohorts of US adults. The American Journal of Clinical Nutrition, 2016; 104(5): 1209-1217. 
7. CHOR D, et al. Prevalence, Awareness, Treatment and Influence of Socioeconomic variables on Control of High Blood Pressure: Results of the ELSA-Brasil Study. Rev. PLoS One, 2015; 10(6): 1-14.

8. COELHO MP, et al. Condições de saúde e trabalho na exploração florestal manual em terrenos montanhosos. Rev. Brasileira de Ergonomia, 2016; 12(1): 335-362.

9. COMMERER MA, et al. The Effects of a Flavonoid-Rich Diet on Oxidative Stress, Inflammation, and Lipid Profile after Elective Percutaneous Coronary Intervention: A Randomized Clinical Trial. Prev. Nutr. Food Sci, 2018; 23 (2): 108114.

10. DRls: Dietary Reference Intakes for calcium and vitamin D. Washington, D.C.: National Academies Press, 2011, 1133p.

11. FRISANCHO AR. New norms of upper limb fat and muscle áreas for assessment of nutritional status. Am J. Clin, 1990; 32:77-97.

12. GARCÍA-ARELLANO A, et al. Dietary Inflammatory Index and Incidence of Cardiovascular Disease in the PREDIMED Study. Nutrients, 2015; 7(6): 4124-38.

13. GAUDEMARIS R, et al. Socioeconomic Inequalities in Hypertension Prevalence and Care the IHPAF Study. Rev. Hypertension, 2002; 39 (6): 1119-1125.

14. IBGE. Pesquisa Nacional em saúde. Percepção de Saúde estilo de vida e doenças crônicas. Brasil grandes regiões e unidades da federação, 2019.

15. IZAR, MCO. Posicionamento sobre o Consumo de Gorduras e Saúde Cardiovascular - 2021. Arq Bras Cardiol; 2021; 116(1):160-212.

16. KHAN K, et al. The effect of viscous soluble fiber on blood pressure: A systematic review and meta-analysis of randomized controlled trials. Nutrition, Metabolism \& Cardiovascular Diseases, 2018; 28: 03-13.

17. KIVIMÄKI M, et al. Long working hours and risk of coronary heart disease and stroke: a systematic review and metaanalysis of published and unpublished data for 603.838 individuals. The Lancet, 2015; 386 (10005):1739-1746.

18. LIMA, TS et al. Perfil nutricional dos trabalhadores do manejo de eucalipto no extremo sul da Bahia. REAS, 2020 Vol.12(3) e 2630

19. LUZ TC, at al. Fatores de risco cardiovascular em uma população rural brasileira. Ciência e saúde coletiva, 2020; 25(10): 3921-3932.

20. MATEO-GALLEGO R, et al. Adherence to a Mediterranean diet is associated with the presence and extension of atherosclerotic plaques in middle-aged asymptomatic adults: The Aragin Workers'Health Study. Journal of Clinical Lipidology, 2017; 11(6): 1372-1382.

21. NASCIMENTO KAO, et al. Frequência cardíaca para estimativas da carga física de trabalho na exploração florestal. biofix Scientific Journal, v.1, n.1, p. 210-215, 2018

22. OPAS. Doenças cardiovasculares - Determinantes Sociais e Riscos para a Saúde, Doenças Crônicas não transmissíveis e Saúde Mental [Internet] Brasília: Organização Pan-Americana da Saúde, 2017.

23. PESCADOR CMM, OLIVEIRA AJ. Segurança do trabalho na colheita florestal: um estudo de caso. Trabalho de Conclusão de Curso (Pós-graduação em Engenharia de Segurança do Trabalho) - Departamento de Engenharia Civil, Setor de Ciências Agrárias e Tecnológicas, Universidade Estadual de Ponta Grossa. Paraná 2009. 60p.

24. PICON RV, et al. Prevalence of hypertension among elderly persons in urban Brazil: a systematic review with metaanalysis. Am. J. Hypertens., 2013; 6(4):541-548.

25. PORTO AS, et al. Consumo de sódio e potássio por diferentes métodos de avaliação: uma revisão em estudos populacionais. Rev. Bras. Pesq. Saúde, 2014; 16(3): 131-139.

26. PRECOMA DB. et al. Atualização da Diretriz de Prevenção Cardiovascular da Sociedade Brasileira de Cardiologia 2019. Arq Bras Cardiol. 2019; 113 (4): 787-891.

27. SAMPAIO LR. Lílian Ramos Sampaio, Maria da Conceição Monteiro da Silva, Tatiane Melo de Oliveira e Christiane Ishikawa Ramos. Antropometria, Av. Nutricional, Salvador: EDUFBA; 2012; 158p.

28. SANTOS SFOM, HAKEYAMA K. Processo sustentável de produção de carvão vegetal quanto aos aspectos: ambiental, econômico, social e cultural. Revista Produção, Curitiba, 2012; 22(2): 309-321.

29. SOCIEDADE BRASILEIRA DE CARDIOLOGIA-SBC. 7ª Diretriz Brasileira de Hipertensão Arterial. In: Malachias, MVB (Org). 2016; 107(3): 44-103.

30. SOCIEDADE BRASILEIRA DE CARDIOLOGIA-SBC. 5a Diretriz Brasileira de Dislipidemias e Prevenção da Aterosclerose. In: Xavier, HT (Org). 2013; 107(4): 44-103.

31. SCHETTINO S, et al. Avaliação ergonômica do processo de mensuração florestal. Rev. Scientia Forestalis. Piracicaba, 2016; 44(111): 575-586.

32. SMITH P, et al. The Relationship Between Occupational Standing and Sitting and Incident Heart Disease Over a 12Year Period in Ontario, Canada. American Journal Of Epidemiology, 2017; 187(1): 27-33.

33. SOUZA EVA, et al. Avaliação da obesidade de trabalhadores rurais em um município do interior da Bahia. Revista Brasileira de Obesidade Nutrição e Emagrecimento, 2012; 6 (31): 69-76.

34. WADA K, et al. Differences in stroke and ischemic heart disease mortality by occupation and industry among Japanese working-aged men. Ssm - Population Health, 2016; 2:745-749.

35. YUN M, et al. Tobacco smoking strengthens the association of elevated blood pressure with arterial stiffness: the Bogalusa Heart Study. J Hypertens, 2015; 33 (2): 266-74. 